\title{
Ultrasound-guided internal jugular vein cannulation: techniques to confirm guidewire location
}

\author{
Praveen Maheshwari, MD • Parul Maheshwari, MD
}

Received: 21 July 2015/Accepted: 17 August 2015/Published online: 26 August 2015

(C) Canadian Anesthesiologists' Society 2015

\section{To the Editor,}

We read with interest the article by Aoyama et al. ${ }^{1}$ regarding a novel technique to confirm the location of guidewires, and we have a few suggestions to supplement correct internal jugular venous (IJV) cannulation and catheter placement.

We agree with the authors' observation that the guidewire may not always be visible during ultrasoundguided IJV cannulation ${ }^{2}$ and commend them for the novel technique they devised to identify guidewire location. We practice several maneuvers at our own institution to assist with identification of the guidewire during central venous cannulation. We perform these maneuvers either alone or in sequence, especially if the guidewire is difficult to visualize.

One of the maneuvers involves placing the ultrasound probe either in plane or obliquely over the guidewire to assist with guidewire identification. It is advantageous to hold the ultrasound probe in line because a significant length of the guidewire can be visualized in the vein, the guidewire's location in the internal jugular vein can be confirmed, and distal arterial cannulation by the guidewire, if any, can be detected. A second maneuver we use to assist with identification of the guidewire in the internal jugular vein involves wiggling the guidewire while it is held close to the entry point in the skin with the ultrasound probe in a transverse position. Our third maneuver involves gradually increasing the pressure exerted by the ultrasound probe over the vein until the vein collapses. When this is followed by the sudden release of the probe, it leads to changes in the intraluminal pressure and movement of the guidewire within the lumen of the vein, thus assisting in its identification.

We suggest using multiple techniques to confirm guidewire location in the internal jugular vein, especially in cases of difficult visualization. This will minimize complications and improve patient safety.

Conflicts of interest None declared.

\section{References}

1. Aoyama K, Takenaka I, Iwagaki T, Sano H. A simple maneuver for confirmation of the guidewire during ultrasound-guided internal jugular vein cannulation. Can J Anesth 2015; 62: 839-40.

2. Moak JH, Lyons MS, Wright SW, Lindsell CJ. Needle and guidewire visualization in ultrasound-guided internal jugular vein cannulation. Am J Emerg Med 2011; 29: 432-6.

This letter is accompanied by a reply. Please see Can J Anesth 2015; 62: this issue.

P. Maheshwari, MD $(\varangle) \cdot$ P. Maheshwari, MD

University of Oklahoma Health Sciences Centre, Oklahoma

City, OK, USA

e-mail: praveen-maheshwari@ouhsc.edu 